\title{
Role of novel antiresorptive agents for the prevention and treatment of osteoporosis
}

\author{
Christoph A Meier
}

Division d'Endocrinologie et Diabétologie et Clinique de Médecine II, Département de Médecine Interne, Hôpital Cantonal Universitaire de Genève, CH-1211 Geneva, Switzerland

Osteoporosis is a significant source of morbidity and mortality in industrialized nations enjoying the benefits of an extended life span. Besides drug treatment (e.g. corticoids, antiepileptics, anticoagulants) and specific, but quite rare, endocrine, hematologic or rheumatologic disorders, estrogen deficiency from secondary amenorrhea or after menopause is the most common cause of osteoporosis. Although physical activity, adequate calcium (1-1.5 g/day) and vitamin D (800 U/day) intake, as well as avoidance of tobacco and alcohol abuse, are important protective factors, they frequently prove insufficient to prevent the progressive bone loss in estrogen-deficient women. Hence, intense pharmaceutical efforts have been aimed at identifying and developing novel compounds for the prevention and treatment of osteoporosis.

The ideal drug would induce the formation of new bone, resulting in a progressive increase in bone mass during the treatment period, such as has been described with fluoride or intermittent parathyroid hormone. However, these two therapeutic modalities are either associated with poor bone quality, or are still in an experimental stage, respectively. On the other hand, several agents are available which inhibit bone resorption, thereby delaying the rate of bone loss and resulting in an initial increase in bone mass during the first 1-3 years of treatment. This latter category encompasses estrogen and its analogs, as well as biphosphonates and calcitonin. However, these hormones and drugs are not without problems, since estrogen increases the risk for certain malignancies, and the older biphosphonates have to be taken intermittently in order to avoid the occurrence of a bone mineralization defect. In addition, non-estrogen based pharmacological approaches to the prevention and treatment of osteoporosis have additional disadvantages. In particular, they do not usually confer the cardioprotective effect of estrogens, which is an important factor in the decreased mortality observed in women taking hormone-replacement therapy. Recent studies with the selective estrogen receptor modulator raloxifene, as well as with the potent biphosphonate alendronate, which lacks the propensity to induce a mineralization defect when taken continuously, have now established their usefulness in the prevention of bone loss in postmenopausal women.

The non-steroidal drug raloxifene is the second drug of the class of tissue-specific estrogen receptor agonists to be used clinically. The first such substance, tamoxifen, has been used extensively for its antiestrogenic properties in women with breast cancer, but its agonistic effect on the endometrium has prevented its wider use in other diseases. Raloxifene, in contrast, is an estrogen antagonist in breast and endometrium, while it retains the estrogen-like activity on bone and lipid metabolism, at least in rodents. Delmas et al. (1) have now conducted the first large placebo-controlled human trial in over 600 postmenopausal women over 24 months, assessing the effects of raloxifene on bone mineral density (BMD), serum lipids and endometrial thickness. The included women were 45-60 years of age and an average of 4-5 years postmenopause; they had a BMD between -2.5 and +2 s.D. compared with young healthy women $(t$-score). After 24 months on $60 \mathrm{mg}$ raloxifene per day, the participating women had an average $2.4 \%$ increase in their lumbar and femoral BMD compared with the women receiving placebo. In addition, serum low density lipoprotein (LDL) cholesterol levels decreased by a median of $10 \%$ on the same dose of raloxifene, while a reduction of merely $1.2 \%$ was noted in the placebo group. In contrast, no significant differences in high density lipoprotein or triglyceride levels were found, and, most importantly, endometrial thickness, as well as the incidence of hot flashes, breast pain and vaginal bleeding, was similar in the placebo and raloxifene groups. In addition, preliminary data suggest that raloxifene possibly decreases the risk for breast cancer.

While these results are very promising, a few caveats need to be considered. First, this study assessed BMD as a surrogate marker for fracture rates. However, the data even for estrogen linking its beneficial effects on BMD to fracture rates are quite scant (2). Hence, it will be crucial to demonstrate the effectiveness of raloxifene in the prevention of hip and/or vertebral fractures. In addition, the cardioprotective effect of raloxifene needs to be established before it can be considered as an alternative for women eligible for standard hormonereplacement therapy. While the LDL-lowering effect of raloxifene is certainly promising, estrogen appears to have additional actions on vasodilatation, possibly contributing to its preventive action on coronary heart disease. It is currently unclear whether raloxifene shares these properties with estrogen. Finally, raloxifene appears to be more than just a tissue-specific estrogen agonist. Studies aimed at elucidating the molecular details of raloxifene's action have shown that the raloxifene-occupied estrogen receptor binds and 
activates novel DNA sequences, not recognized by the native estradiol-occupied receptor. However, whether this signaling pathway is pharmacologically relevant, and whether it mediates beneficial or potentially adverse effects of this novel drug remain to be elucidated.

Until the cardioprotective effects of raloxifene are established, its use is likely to be restricted to women with contraindications for the use of classical estrogenreplacement therapy. However, the novel biphosphonate alendronate is also a candidate drug for this indication. In a placebo-controlled trial in postmenopausal women with established osteoporosis, alendronate (10 mg/day) was previously shown to increase BMD and reduce fracture rates significantly by $48 \%$ for the spine, while a trend towards a reduction of non-vertebral fractures was also present (3). Hosking et al. (4) have now compared alendronate with placebo and estrogenprogesterone-replacement therapy for the prevention of osteoporosis in nearly 1200 young postmenopausal women (mean age 53 years). The investigators found that the use of $5 \mathrm{mg}$ alendronate per day over 2 years increased lumbar and hip BMD by 3.5 and $1.9 \%$ respectively. This increase in BMD was 1-2 percentage points lower compared with hormone-replacement therapy. Longer term studies are needed, however, to examine whether this apparently lower increase in BMD might simply reflect a slower filling of the remodeling space in the patients treated with alendronate. It is notable that the safety and side-effect profiles of alendronate and placebo were comparable, even with regard to gastrointestinal side-effects.
In summary, these two novel compounds show promise for the prevention of osteoporosis in postmenopausal women. The decision to use either drug in postmenopausal women with contraindications (or an unwillingness) to take estrogens requires an informed individualized decision. Although alendronate has been proven effective in preventing vertebral fractures in women with established osteoporosis, it is cumbersome to ingest (the drug needs to be taken on an empty stomach, and requires remaining in an upright position for 30 min) and lacks the cardioprotective effects of estrogen. The benefits of raloxifene (lipid lowering, antiestrogenic action on breast and uterus) beyond its action on bone resorption are very attractive, but the potentially beneficial effects of this novel drug on cardiovascular events and fractures are not yet established.

\section{References}

1 Delmas PD, Bjarnason NH, Mitlak BH, Ravoux AC, Shah AS, Huster WJ et al. Effects of raloxifene on bone mineral density, serum cholesterol concentrations, and uterine endometrium in postmenopausal women. New England Journal of Medicine 1997337 1641-1647.

2 Eastell R. Treatment of postmenopausal osteoporosis. New England Journal of Medicine 1998338 736-746.

3 Liberman UA, Weiss SR, Bröll J, Minne HW, Quan H, Bell NH et al. Effect of oral alendronate on bone mineral density and the incidence of fractures in postmenopausal osteoporosis. New England Journal of Medicine 1995333 1437-1443.

4 Hosking D, Chilvers CE, Christiansen C, Ravn P, Wasnich R, Ross P et al. Prevention of bone loss with alendronate in postmenopausal women under 60 years of age. New England Journal of Medicine 1998338 485-492. 